Mesenchymal stem cells (MSCs) are attractive seed cells for immunotherapy, tissue engineering and regenerative medicine due to their self-renew$\mathrm{al}$ and multidirectional differentiation abilities, diverse immunoregulatory functions and ease of isolation from a wide range of tissues. MSCs exert their immunoregulatory effect on immune cells via cell-to-cell contact and paracrine mechanisms. In turn, MSCs can also be modulated by immune cells. Macrophages are constantly present in the mucosa of the intestinal tract of mammals and play an important role in the development and progression of inflammatory bowel disease (IBD), a chronic and recurrent inflammatory disease of the gastrointestinal tract characterized by idiopathic mucosal inflammation. The increased morbidity and mortality of IBD have made it a disease hard to cure in the clinic. MSCs have emerged as an important tool for IBD therapy due to their abilities to differentiate into enterocyte-like cells and regulate inflammatory cells, especially macrophages. In this review, we discuss the recent advances in the interaction between MSCs and macrophages in diseases, with an emphasis on IBD. We propose that an optimized MSC-based therapy would provide a novel strategy for the treatment of IBD and the prevention of IBD-associated colorectal cancer (CRC).

Key words: mesenchymal stem cell, inflammatory bowel disease, macrophage, immunoregulatory, colorectal cancer.

Contemp Oncol (Pozn) 2017; 21 (2): 91-97 DOI: https://doi.org/10.5114/wo.2017.68616

\section{Crosstalk between mesenchymal stem cells and macrophages in inflammatory bowel disease and associated colorectal cancer}

\begin{abstract}
Fei $\mathrm{MaO}^{1^{*}}$, Jing-jing Kang ${ }^{1^{*}}$, Xiu Cai ${ }^{1}$, Ning-feng Ding ${ }^{1}$, Yun-bing $\mathrm{Wu}^{1}$, Yong-min Yan ${ }^{1}$, Hui Qian ${ }^{1}$, Xu Zhang ${ }^{1}$, Wen-rong $X^{1}$
\end{abstract}

${ }^{1}$ Key Laboratory of Medical Science and Laboratory Medicine of Jiangsu Province, School of Medicine, Jiangsu University, Zhenjiang, Jiangsu, P.R. China.

*These authors contributed equally to this work.

\section{Introduction}

In the past decades, stem cell therapies have been emerging as a potential tool to treat diseases. According to the literature, Tavassoli et al. first reported that autologous fragments of rodent bone marrow were able to generate heterotopic marrow and bone upon transplantation in 1968 [1]. The group of Friedenstein successfully identified and cultured a subpopulation of stromal cells from bone marrow, which had a high replicative potential and could form colonies of fibroblastic cells in vitro, displaying the ability of colony forming unit-fibroblast (CFU-F), which could form ectopic bone marrow comprising trabecular bone, chondrocytes, adipocytes, and hematopoiesis-supporting stroma $[2,3]$. These stromal cells were termed osteogenic stem cells or bone marrow stromal stem cells [4, 5]. In 1991, Caplan Al named the CFU-F identified by Friedenstein et al. as MSCs based on the fact that they could behave as unique progenitors for all connective tissues of mesodermal origin [6]. In 2006, the International Society for Cellular Therapy (ISCT) established the unified and minimal criteria to define MSCS [7]. To date, great efforts have been made to study the nature, phenotype, function, and regulation of MSC in health and diseases.

Mesenchymal stem cells are a heterogeneous population of fibroblast-like, plastic-adherent cells with the potential to self-renew and differentiate into distinct types of cell lineages such as osteoblasts, chondrocytes, adipocytes, tenocytes, myotubes, neural cells, and hematopoietic-supporting stroma, either in vitro or in vivo [8-10]. Mesenchymal stem cells can be isolated from various tissues such as bone marrow, umbilical cord, and adipose [11]. In the early 2000s, it was reported that MSCs display an immunomodulatory effect on lymphocytes $[12,13]$. Mesenchymal stem cells have also been shown to regulate the proliferation, differentiation and immune function of myeloid cells ranging from monocytes [14], dendritic cells (DCs) [15], macrophages $[16,17]$, and myeloid-derived suppressor cells (MDSCs) [18], to granulocytes [19]. In recent years, MSCs have been tested in a variety of diseases such as myocardial infarction [20, 21], acute renal failure [22], acute liver injury [23], collagen-induced arthritis (CIA) [24], graft-versus-host disease (GVHD) [25], and IBD [26].

Inflammatory bowel disease, including Crohn's disease (CD) and ulcerative colitis (UC), is a chronic, recurrent inflammatory disease of the gastrointestinal tract and is characterized by idiopathic mucosal inflammation. The incidence and prevalence of IBD are generally higher in the western countries than in Asia, but are rapidly increasing in Asia [27]. Furthermore, $\mathrm{IBD}$ is associated with increased mortality risk as IBD is known to be linked 
to the development of colorectal cancer (colitis-associated colorectal cancer, CAC) [28]. However, there is still no effective method to cure IBD. Recent studies suggest that MSCs are an important therapeutic tool in IBD. MSCs can migrate into intestinal injured tissue and differentiate into enterocyte-like cells $[29,30]$. In addition, MSCs can release growth factors and cytokines to inhibit intestinal inflammation, regulate immune responses and promote enterocyte proliferation to repair the injured tissue [31]. In this review, we discuss the emerging role of MSCs in the treatment of IBD and IBD-associated colorectal cancer.

\section{The immunoregulatory function of mesenchymal} stem cells

In 1986, Juneja et al. reported that MSCs interacted with the transformed cells UTMB-460 (a monoclonal human $\mathrm{B}$-lymphoblastic cell line) in vitro and played a critical role in the establishment of the UTMB-460 cell line, which is the first study about the effect of MSCs on immune cells [32]. Since then, the immunoregulatory functions of MSC have been gradually revealed by the researchers. MSCs are found to inhibit the proliferation of T cells, B cells, and natural killer (NK) cells [33-35]; alter the cytokine secretion profile of dendritic cells (DCs), naive and effector $T$ cells ( $T$ helper $1[T(H) 1]$ and $T(H) 2$ ), and NK cells [35-37]; suppress the migration, maturation and antigen presentation of DCs [38]; restrain the induction of cytotoxic response of T cells and NK cells to alloantigens [35, 39]; and modulate the M1/M2 balance of macrophages [40]. The mechanisms involved in the immunomodulatory roles of MSC include cell-to-cell contact, secretion of regulatory cytokines and other factors, the expression of inhibitory membrane molecules, and induction of cell anergy and apoptosis [41, 42].

\section{Mesenchymal stem cell-mediated immunoregulation on macrophages via cell-to-cell contact}

Cells are known to communicate via secreted molecules and by cell surface molecules, which are deciphered by the target cell upon receptor binding, or by direct cell-tocell contact, mediated by specialized molecules [43]. Mesenchymal stem cells have an immunoregulatory effect on macrophages through direct cell-to-cell contact. Siniscalco et al. demonstrated that MSCs homing to the spinal cord induced the switch of macrophages to an $M 2$ phenotype with anti-inflammatory functions. The intravenously injected MSCs could reduce the protein and mRNA levels of IL-1 $\beta$ and IL-17 and up-regulated the expression of IL-10 protein, which suppresses the proliferation and activation of T cells in the spinal cord of SNI mice [44]. The mechanism for the modulation of macrophages by MSCs to shift from pro-inflammatory to anti-inflammatory phenotype still remains unknown. The administration of MSCs exerts long-term therapeutic efficacy, suggesting that the immunomodulatory properties of MSCs contribute to tissue repair and regeneration. Gur-Wahnon et al. reported that MSCs suppress antigen-presenting cell (APC) maturation by a novel mechanism involving cell-cell interaction rather than the classical mechanism of induction by cytokines $[45,46]$.
Indeed, although MSCs could home to the site of damaged tissue, they have poor survival ability and transitory persistence in the inflammatory area. MSCS are multipotent stem cells that can modulate immune cells such as regulatory $T$ cells to improve immune dysfunction, so these cells are mainly used for the treatment of immune disorders, such as IBD. Intraperitoneal injections of MSCS can markedly reduce intestinal inflammation [47]. In IBD, the mechanism for the regulation of macrophages by MSCs is complicated and various, and mainly involves cell-to-cell contact and paracrine effects. MSCs have the capacity for migration to colon tissues and inhibition of colitis and the potential for suppression of the development of CAC.

\section{Mesenchymal stem cell-mediated immunoregulation of macrophages via paracrine actions}

Mesenchymal stem cells transplantation maintains the survival of monocytes, alters the macrophage phenotype from M1 to M2 in the damaged tissue or organ, such as spleen and bone marrow, and exerts therapeutic effects on inflammatory disease remission via the paracrine effect. Mesenchymal stem cells have poor survival ability and transitory persistence in the inflammatory area. Mesenchymal stem cell transplantation into the injured tissues might be involved in paracrine action by secretion of soluble factors [48]. Paracrine is a kind of cellular communication in which a cell produces a molecule to induce changes in the adjacent cells, altering the behavior or differentiation of those cells. Jin et al. found that deregulation of inflammatory soluble factors such as IL-1 $\alpha$, IL-6, and IL-8 via angiopoietin-1 (Ang-1) could be treated as a potential soluble factor in the co-culture of MSCs with LPS-treated alveolar macrophages. These results indicated that MSCS exerted paracrine actions by increasing the proportion of anti-inflammatory macrophages (M2) and stimulating the production of anti-inflammatory factors in the damaged tissue [49]. The mechanism by which MSCs promote regulatory $T$ cell production is explored as the primary reason for MSC cellular therapy. Induction of MSCS to regulatory T cell generation through TGF- $\beta 1$ secreted by MSCs is dependent on the paracrine production of CCL-18 between MSCs and monocytes or macrophages [50].

Mesenchymal stem cell administration induces B220+CD11b+ monocytes/macrophages and attenuates corneal allograft and experimental autoimmune uveitis (EAU) [51]. Principle mechanisms of tissue repair of MSCS are based primarily on their paracrine effect through expressing soluble factors. Actually, different kinds of MSC paracrine actions are applied to alleviate inflammation such as trophic, immunomodulatory, anti-scarring, and chemoattractant. Recently, $\mathrm{Ti}$ et al. reported that the therapeutic efficiency of LPS-preprocessing MSC-induced exosomes was valuable to wound healing and chronic inflammation, which indicated that MSCs might secrete an amount of exosomes to enhance the trophic effect through a paracrine action [52]. Almeida et al. also showed that MSCs expressed a high level of trophic factors to improve the function of the injured spinal cord [53]. Trans- 
plantation of membrane-derived MSCs remarkably deregulated glomerular monocytes/macrophages infiltration and expression of glomerular inflammatory factors such as TNF- $\alpha$, monocyte chemoattractant protein 1 (MCP-1), type I collagen, and TGF- $\beta$, contributing to the acute renal injury healing [54].

Mesenchymal stem cells co-cultured with macrophages secrete more immunomodulatory factors and express fewer pro-inflammatory factors such as prostaglandin E2 (PGE2) and indoleamine 2,3-dioxygenase (IDO), which can be further enhanced by interferon $\gamma($ IFN- $\gamma)$ or tumor necrosis factor $\alpha$ (TNF- $\alpha$ ) [55]. The immunomodulatory function of MSCs can also be mediated through several signaling pathways. Activation of Toll-like receptors (TLRs), CSF-2 and CSF-3 could modulate the TLR-2/6-induced MT1-MMP/ JAK/STAT3 signaling pathway targeted with neovascularization, resulting in an immunomodulatory effect of MSCs [56]. Mesenchymal stem cells can exert an immunomodulatory effect on macrophages via cell-to-cell contact and paracrine actions, and macrophages also modulate MSCs to a certain extent.

\section{Macrophage-mesenchymal stem cell relations}

Cell-to-cell contact and paracrine actions by MSCs are recognized as the primary mechanisms in immunomodulation so plenty of evidence has been obtained in order to review the effects of MSC-mediated immunomodulation on monocytes/macrophages. However, the mutual relation between MSCs and monocytes/macrophages has gradually gained recognition in recent research studies. Monocytes/macrophages also can regulate MSCs to repair damaged tissue and exert their anti-inflammatory function. Gong et al. reported that M2 macrophages co-cultured with MSCs could promote the expression of ALP, osteogenic markers, and bone mineralization to sustain bone and expedite bone repair [57]. Macrophages, but not M1 macrophages, can promote MSC osteoblast differen- tiation [58]. Sesia et al. also reported that in the collagen scaffolds, MSCs co-cultured with macrophages (M2 polarization), which stem from monocytes, existed a stronger clonogenic and chondrogenic competence contributing to cartilage formation [59]. Such similar interaction also occurs in cardiac disease. Macrophage depletion destroyed the infarct healing and repair in the early therapy, but $M 2$ macrophages (F4/80(+) CD206(+)) incubated with or without MSCs had a positive effect on infarct size and left ventricular remodeling [60]. LPS-treated macrophages co-cultured with MSCs expressed less TNF- $\alpha$ and IL-1 and more IL-10 in the atherosclerotic plaque [61].

Although the above evidence supports the hypothesis that macrophages have the capacity to regulate MSC immunomodulation, the specific mechanism of macrophages acting on MSCs is still vague. However, the following example may provide some insight. In a mouse asthma model, M2 macrophages devoured MSCs while M1 macrophages did not exhibit the phenomenon, indicating that lung macrophages devoured MSCs to remit the allergic asthma [62]. MSC-derived cell-to-cell contact and paracrine actions are of vital importance to suppress inflammation and protect and repair damaged tissues, but the role of macrophages in MSC action should not be underestimated in research work of the correlativity between MSCs and macrophages.

\section{Mesenchymal stem cells regulate macrophages to treat inflammatory bowel disease}

Mesenchymal stem cells-mediated immunoregulation of macrophages via paracrine actions has been demonstrated in various inflammatory diseases, such as bone repair, wound healing, myocardial infarction, acute kidney injury, atherosclerosis, and IBD. We searched the PubMed database using the terms "MSC and macrophage". There are a total of 530 documents published since 1976 and they have increased rapidly in recent years (Fig. 1). Activated
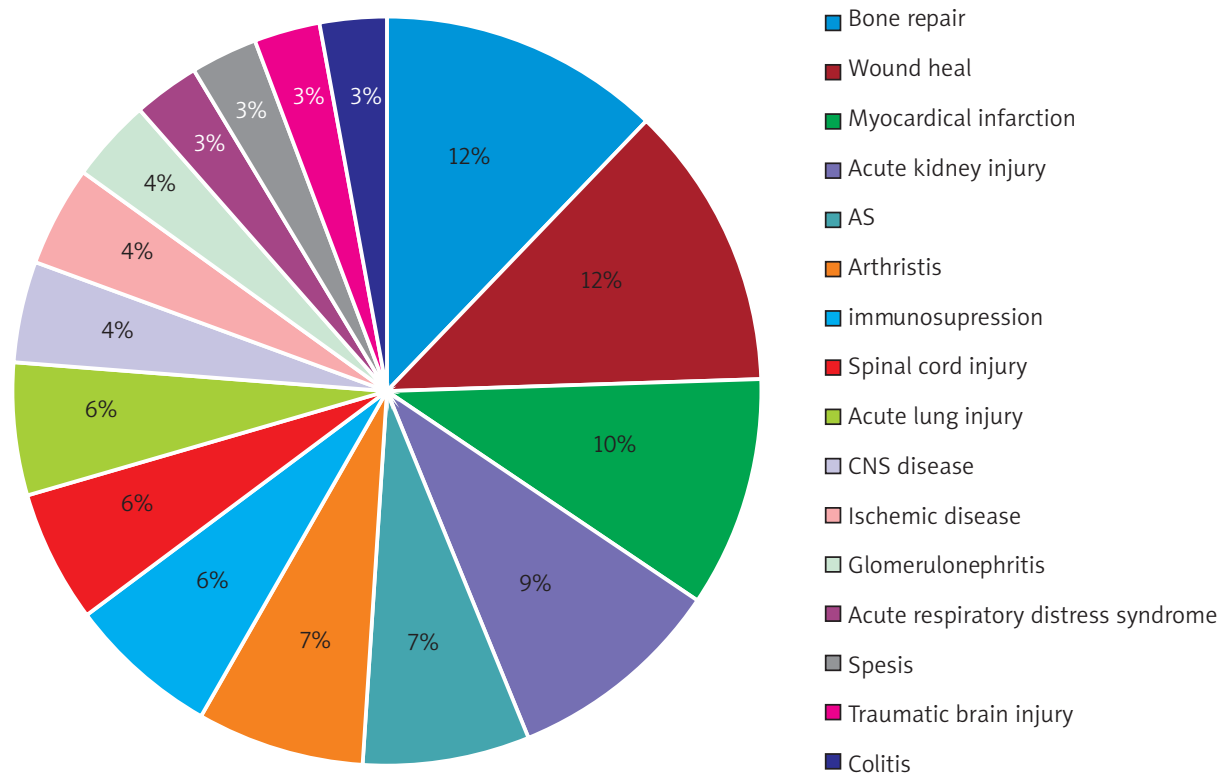

Fig. 1. Distribution of MSC regulation on macrophages in different diseases 
MSCs stimulate alternatively macrophage activity to boost wound tensile strength in senior mice [63]. In the infarction location, pro-inflammatory macrophages were gradually transformed to anti-inflammatory macrophages via the increased expression of IL-10 mediated by MSCs to ameliorate acute myocardial infarction [64]. Fernandes et al. reported that osteoclasts and macrophages can promote the process of bone formation whereby MSCs stimulate macrophages (M2) dependent on gp130 or oncostatin M [58].

Inflammatory bowel disease is a type of idiopathic intestinal tract disease involving the ileum, rectum and colon, while various anti-inflammatory and immunosuppressive therapy, such as corticosteroids, immunosuppressive agents and biological agents, do not have a longterm clinical curative effect on IBD, especially refractory IBD [65]. Cell therapy research on MSCs associated with $\mathrm{IBD}$ has emerged continuously in recent years because of MSC immunosuppressive capability [66]. Although MSCs can home to the damaged intestinal mucosa, the short duration in the resting place requires repeated treatment during each phase of clinical treatment [67]. More functions of MSCS for the treatment of IBD are obtained through paracrine soluble molecules and regulating $T$ cell number and function [68-70]. MSCs can also boost the production of Treg cells in the intestinal inflammatory region, thereby reducing the antigenic effect of the antigen and debasing the inflammatory factor and enhancing immune cell apoptosis [71].

The salutary effect of MSC on IBD is also dependent on the dose and the mode of MSC infusion. In in vivo experiments, the dose of MSC administration is usually $10^{5} \sim 10^{6}$ MSCs/mouse [72] and in pre-clinical trials, the amount of administration is usually about $10^{6} \sim 10^{7} / \mathrm{KG}$, and the therapeutic effect may increase with the dose to some extent, but the side effects caused by different doses are still not fully described [73]. For perianal fistulizing CD, in both in vivo and pre-clinical trials, the curative effect of perianal injection and intraperitoneal injection of MSC is better than intravenous MSC, as judged through the colon length, weight loss control and clinical pathology score. In contrast, for luminal IBD, the efficacy of intravenous injection is also weaker than local MSC injection. Ko et al. demonstrated that MSCs coated with antibodies and delivered to the colon, which modified the survival rates, therapeutic scores and body weight in the IBD mice [74]. Also researchers have detected that MSCs not only have the efficiency to upgrade the length of the colon and size of the spleen but also depress the risk of colorectal cancer via reducing the production of TNF- $\alpha, \mathrm{IL}-1 \beta$ and IL- 6 and stimulation of the STAT3 signaling pathway [75]. The therapeutic effects of MSCs with intraperitoneal injection and IBD drugs are mutually compatible [76]. The gene expression level in IBD mice treated with MSCs is also discrepant compared to $I B D$ rats and the normal group. Xing et al. established IBD rat models to verify such a hypothesis. The results demonstrated that in the MSC-treated group, the expression of the genes Olfm4 and Wnt3a was deregulated while GSK$3 \beta$ was upregulated, which contrasts with the IBD group [77]. Wang et al. also discovered that MSC administration decreased the expression of IL-31b [78]. Although such benefits derived from MSC treatment are able to relieve the pain of patients with IBD, there are numerous difficulties involved in the application of MSCS. For example, the IBD animal model cannot completely replace and reflect the actual condition of IBD patients. Different sources of MSCs can also differentially influence the MSC action [79].

Macrophages play a crucial role in the pathogenesis of IBD stimulated by intestinal microorganism [80, 81]. CD68+ macrophages accumulated in the lamina of the intestinal inflammatory lesions reduce the expression of tight junction proteins, and damage epithelial cell barrier function and integrity, thereby accelerating disease progression via stimulating iNOS and TNF- $\alpha$ expression [82]. MSCs influence the balance between M1 and M2 macrophages to exert a therapeutic effect. Wang et al. reported that in the acute colitis mouse model, the clinical pathological score was significantly decreased in the MSC group compared with the control group. M2 macrophage polarization was enhanced significantly in DSS-colitis with MSC administration and the TGF- $\beta$ signaling pathway was stimulated mainly by anti-inflammatory macrophages, while the therapeutic effect of MSCs on IBD would fade away if the TGF- $\beta$ signaling pathway was inhibited [83]. In the peritoneal cavity of mice with acute colitis, the amount of CD206+ M2 macrophages was significantly increased by MSCs with IL-1 pretreatment, which inhibited the number of $\mathrm{CD} 11 \mathrm{c}+\mathrm{M} 1$ macrophages, thus alleviating inflammatory responses in the intestinal mucosa [84]. Semitic et al. also demonstrated that MSCs mediated the alternative activation of macrophages through the inhibition of galectin-3 [85]. We have demonstrated that hucMSCs could repair DSS-induced IBD through the regulation of 15-LOX-1 expression and the modulation of inflammatory responses in macrophages [86]. The mechanism of MSC regulation of macrophages to treat IBD remains vague, and more intensive and pervasive investigations are needed to better understand it.

\section{Mesenchymal stem cells regulate macrophages to treat inflammatory bowel disease-associated colorectal cancer}

Ulcerative colitis (UC) and Crohn's disease (CD) are associated with an increased rate of colorectal cancer (CRC) $[87,88]$. The estimated risk of CRC varies among studies, although it is accepted that the risk for malignancy increases with duration of diagnosis $[89,90]$. CRC is the third most commonly diagnosed cancer worldwide, which develops slowly and begins with adenoma and progresses over several years to carcinoma [91, 92]. The present studies indicate that MSCs have an effect on the treatment of CRC. The stromal cell niche in the intestine is comprised of numerous, heterogeneous subsets of stromal cells, which include CD45-EpCAM-cells, fibroblasts, myofibroblast, and MSCs [93]. Chen et al. reported that MSCs not only could increase the length of the colon and the size of the spleen but also could reduce the risk of colorectal cancer via reducing the production of TNF- $\alpha, I L-1 \beta$ and IL- 6 and inhibiting activation of the STAT3 signaling pathway [75]. 
Evidence shows that macrophages in the tumor microenvironment display M2 phenotype, which dampens the anti-tumor immune responses [94-96]. MSCs can exert their tumor protecting effects by interacting with macrophages in the tumor microenvironment $[94,95]$. Mesenchymal stem cells can polarize macrophages towards the "anti-inflammatory" M2 phenotype, characterized by increased IL-10 production and decreased iNOS and IL-12 expression [94, 95, 97, 98]. However, Sala et al. reported that MSCs transplanted into mice with CRC by intraperitoneal injection did not completely home to the damaged inflammatory intestinal mucosa lesion, but rather accumulated in the abdominal cavity and were accompanied by T cells and macrophages. Macrophages treated with MSCs show M2 phenotype with increased production of IL-10 and iNOS and decreased expression of IFN- $\gamma$, IL- 6 and TNF [47].

\section{Conclusions}

In this review, we elaborate the immunoregulatory effect of MSCs on macrophages via cell-to-cell contact and paracrine actions, which inhibits the secretion of pro-inflammatory factors in macrophages to alleviate the severity of IBD. Macrophages also have the capacity to regulate MSCS, which still needs more in-depth analyses to determine the specific mechanism. It seems that MSCs from different sources may have distinct therapeutic efficacy according to the disease. It is necessary to choose the proper approach for MSC transplantation for different types of IBD. The dose of injected MSC also needs to be optimized, which is critical to the repair of damaged intestinal mucosa. Finally, the mechanism for the potential modulation of macrophages by MSCs in IBD needs to be further explored. If these problems were satisfactorily solved, MSC-based therapy would provide a novel strategy for the treatment of IBD and the prevention of IBD-associated CRC.

This study was funded by the National Natural Science Foundation of China (Grant no. 81670502, 81672416, 81272481), Jiangsu Key Research and Development Project (Grant no. BE2016717), Jiangsu Province for Outstanding Sci-tech Innovation Team in Colleges and Universities (SJK2013-10), the China postdoctoral science foundation (Grant no. 2015M571705, 2015M580403 and 2016T90431), the Scientific Research Foundation of Jiangsu University (Grant no. FCJJ2015023), the opening project of the Key Laboratory of Embryo Molecular Biology, Ministry of Health of China, and Shanghai Key Laboratory of Embryo and Reproduction Engineering (Grant no. KF201601), and Project Funded by the Priority Academic Program Development of Jiangsu Higher Education Institutions.

The authors declare no conflict of interest.

\section{References}

1. Tavassoli M, Crosby WH. Transplantation of marrow to extramed ullary sites. Science 1968; 161: 54-6.

2. Friedenstein AJ, Chailakhjan RK, Lalykina KS. The development of fibroblast colonies in monolayer cultures of guinea-pig bone marrow and spleen cells. Cell Tissue Kinet 1970; 3: 393-403.
3. Friedenstein AJ, Chailakhyan RK, Latsinik NV, et al. Stromal cells responsible for transferring the microenvironment of the hemopoietic tissues. Cloning in vitro and retransplantation in vivo. Transplantation 1974; 17: 331-40.

4. Friedenstein AJ, Chailakhyan RK, Gerasimov UV. Bone marrow osteogenic stem cells: in vitro cultivation and transplantation in diffusion chambers. Cell Tissue Kinet 1987; 20: 263-72.

5. Owen M, Friedenstein AJ. Stromal stem cells: marrow-derived osteogenic precursors. Ciba Found Symp 1988; 136: 42-60.

6. Caplan Al. Mesenchymal stem cells. J Orthop Res 1991; 9: 641-50.

7. Dominici M, Le Blanc K, Mueller I, et al. Minimal criteria for defining multipotent mesenchymal stromal cells. The International Society for Cellular Therapy position statement. Cytotherapy 2006; 8: 315-7.

8. Tse WT, Pendleton JD, Beyer WM, et al. Suppression of allogeneic T-cell proliferation by human marrow stromal cells: implications in transplantation. Transplantation 2003; 75: 389-97.

9. Pittenger MF, Mackay AM, Beck SC, et al. Multi-lineage potential of adult human mesenchymal stem cells. Science 1999; 284: 143-7.

10. Deans RJ, Moseley AB. Mesenchymal stem cells: biology and potential clinical uses. Exp Hematol 2000; 28: 875-84.

11. Ti D, Hao H, Fu X, et al. Mesenchymal stem cells derived exosomal microRNAs contribute to wound inflammation. Sci China Life Sci 2016; 59: 1305-12.

12. Bartholomew A, Sturgeon C, Siatskas M, et al. Mesenchymal stem cells suppress lymphocyte proliferation in vitro and prolong skin graft survival in vivo. Exp Hematol 2002; 30: 42-8.

13. Di Nicola M, Carlo-Stella C, Magni M, et al. Human bone marrow stromal cells suppress T-lymphocyte proliferation induced by cellular or nonspecific mitogenic stimuli. Blood 2002; 99: 3838-43.

14. Chen PM, Liu KJ, Hsu PJ, et al. Induction of immunomodulatory monocytes by human mesenchymal stem cell-derived hepatocyte growth factor through ERK1/2. J Leukoc Biol 2014; 96: 295-303.

15. Chiesa S, Morbelli S, Morando S, et al. Mesenchymal stem cells impair in vivo T-cell priming by dendritic cells. Proc Natl Acad Sci U S A 2011; 108: 17384-9.

16. Nemeth K, Leelahavanichkul A, Yuen PS, et al. Bone marrow stromal cells attenuate sepsis via prostaglandin E (2)-dependent reprogramming of host macrophages to increase their interleukin-10 production. Nat Med 2009; 15: 42-9.

17. Oh JY, Ko JH, Lee HJ, et al. Mesenchymal stem/stromal cells inhibit the NLRP3 inflammasome by decreasing mitochondrial reactive oxygen species. Stem Cells 2014; 32: 1553-63.

18. Yen BL, Yen ML, Hsu PJ, et al. Multipotent human mesenchymal stromal cells mediate expansion of myeloid-derived suppressor cells via hepatocyte growth factor/c-met and STAT3. Stem Cell Reports 2013; 1: 139-51.

19. Chen CP, Chen YY, Huang JP, et al. The effect of conditioned medium derived from human placental multipotent mesenchymal stromal cells on neutrophils: possible implications for placental infection. Mol Hum Reprod 2014; 20: 1117-25.

20. He H, Zhao ZH, Han FS, et al. Overexpression of protein kinase C $\varepsilon$ improves retention and survival of transplanted mesenchymal stem cells in rat acute myocardial infarction. Cell Death Dis 2016; 7: e2056.

21. Qian H, Yang H, Xu W, et al. Bone marrow mesenchymal stem cells ameliorate rat acute renal failure by differentiation into renal tubular epithelial-like cells. Int J Mol Med 2008; 22: 325-32.

22. Musialek P, Mazurek A, Jarocha D, et al. Myocardial regeneration strategy using Wharton's jelly mesenchymal stem cells as an offthe-shelf 'unlimited' therapeutic agent: results from the Acute Myocardial Infarction First-in-Man Study. Postepy Kardiol Interwencyjnej 2015; 11: 100-7.

23. Fu J, Zhang H, Zhuang Y, et al. The role of $\mathrm{N}$-acetyltransferase 8 in mesenchymal stem cell-based therapy for liver ischemia/reperfusion injury in rats. PLoS One 2014; 9: e103355.

24. Mao F, Xu WR, Qian H, et al. Immunosuppressive effects of mesenchymal stem cells in collagen-induced mouse arthritis. Inflamm Res 2010; 59:219-25.

25. Polchert D, Sobinsky J, Douglas GW, et al. IFN-gamma activation of mesenchymal stem cells for treatment and prevention of graft versus host disease. Eur J Immunol 2008; 38: 1745-55. 
26. Abdel Salam AG, Ata HM, Salman TM, et al. Potential therapeutic utility of mesenchymal stem cells in inflammatory bowel disease in mice. Int Immunopharmacol 2014; 22: 515-21.

27. Prideaux L, Kamm MA, De Cruz PP, et al. Inflammatory bowel dis ease in Asia: a systematic review. J Gastroenterol Hepatol 2012; 27: 1266-80.

28. Ahmadi A, Polyak S, Draganov PV. Colorectal cancer surveillance in inflammatory bowel disease: the search continues. World J Gastroenterol 2009; 15: 61-6.

29. Tanaka F, Tominaga K, Ochi M, et al. Exogenous administration of mesenchymal stem cells ameliorates dextran sulfate sodiuminduced colitis via anti-inflammatory action in damaged tissue in rats. Life Sci 2008; 83: 771-9.

30. Yabana T, Arimura Y, Tanaka H, et al. Enhancing epithelial engraftment of rat mesenchymal stem cells restores epithelial barrier integrity. J Pathol 2009; 218: 350-9.

31. Watanabe S, Arimura Y, Nagaishi K, et al. Conditioned mesenchymal stem cells produce pleiotropic gut trophic factors. J Gastroenterol 2014; 49: 270-82.

32. Juneja HS, Rajaraman S, Ramsey KM, et al. Role of marrow stromal cells in the establishment of a transformed lymphoblastic B-cell line from a normal human subject. Leuk Res 1986; 10: 1209-19.

33. Di Nicola M, Carlo-Stella C, Magni M, et al. Human bone marrow stromal cells suppress T-lymphocyte proliferation induced by cellular or nonspecific mitogenic stimuli. Blood 2002; 99: 3838-43.

34. Le Blanc K, Ringdén O. Immunomodulation by mesenchymal stem cells and clinical experience. J Intern Med 2007; 262: 509-25.

35. Spaggiari GM, Capobianco A, Abdelrazik H, et al. Mesenchymal stem cells inhibit natural killer-cell proliferation, cytotoxicity, and cytokine production: role of indoleamine 2, 3-dioxygenase and prostaglandin E2. Blood 2008; 111: 1327-33.

36. Aggarwal S, Pittenger MF. Human mesenchymal stem cells modulate allogeneic immune cell responses. Blood 2005; 105: 1815-22.

37. Abumaree M, Al Jumah M, Pace RA, et al. Immunosuppressive properties of mesenchymal stem cells. Stem Cell Rev 2012; 8: 375-92.

38. English K, Barry FP, Mahon BP. Murine mesenchymal stem cells suppress dendritic cell migration, maturation and antigen presentation. Immunol Lett 2008; 115: 50-8.

39. Angoulvant D, Clerc A, Benchalal S, et al. Human mesenchymal stem cells suppress induction of cytotoxic response to alloantigens. Biorheology 2004; 41: 469-76.

40. Cho DI, Kim MR, Jeong HY, et al. Mesenchymal stem cells reciprocally regulate the $\mathrm{M} 1 / \mathrm{M} 2$ balance in mouse bone marrow-derived macrophages. Exp Mol Med 2014; 46:e70.

41. Holan V, Hermankova B, Bohacova P, et al. Distinct immunoregulatory mechanisms in mesenchymal stem cells: role of the cytokine environment. Stem Cell Rev 2016; 12: 654-63.

42. English K. Mechanisms of mesenchymal stromal cell immunomod ulation. Immunol Cell Biol 2013; 91: 19-26.

43. Turturici G, Tinnirello R, Sconzo G, et al. Extracellular membrane vesicles as a mechanism of cell-to-cell communication: ad vantages and disadvantages. Am J Physiol Cell Physiol 2014; 306: c621-33.

44. Siniscalco D, Giordano C, Galderisi U, et al. Long-lasting effects of human mesenchymal stem cell systemic administration on painlike behaviors, cellular, and biomolecular modifications in neuropathic mice. Front Integr Neurosci 2011; 5: 79.

45. Gur-Wahnon D1, Borovsky Z, Beyth S, et al. Contact-dependent induction of regulatory antigen-presenting cells by human mes enchymal stem cells is mediated via STAT3 signaling. Exp Hematol 2007; 35:426-33.

46. Gur-Wahnon D, Borovsky Z, Liebergall M, et al. The induction of APC with a distinct tolerogenic phenotype via contact-dependent STAT3 activation. PLoS One 2009; 4:e6846.

47. Sala E, Genua M, Petti L, et al. Mesenchymal stem cells reduce colitis in mice via release of TSG6, independently of their localization to the intestine. Gastroenterology 2015; 149: 163-76.

48. Gnecchi M, Danieli P, Malpasso G, et al. Paracrine mechanisms of mesenchymal stem cells in tissue repair. Methods Mol Biol 2016; 1416: $123-46$

49. Jin HJ, Bae YK, Kim M, et al. Comparative analysis of human mesenchymal stem cells from bone marrow, adipose tissue, and um- bilical cord blood as sources of cell therapy. Int J Mol Sci, 2013; 14: 17986-8001.

50. Melief SM, Schrama E, Brugman MH, et al. Multipotent stromal cells induce human regulatory $T$ cells through a novel pathway involving skewing of monocytes toward anti-inflammatory macrophages. Stem Cells 2013; 31: 1980-91.

51. Ko JH, Lee HJ, Jeong HJ, et al. Mesenchymal stem/stromal cells precondition lung monocytes/macrophages to produce tolerance against allo- and autoimmunity in the eye. Proc Natl Acad Sci U S A 2016; 113: 158-63.

52. Ti D, Hao H, Tong C, et al. LPS-preconditioned mesenchymal stromal cells modify macrophage polarization for resolution of chronic inflammation via exosome-shuttled let-7b. J Transl Med 2015; 13: 308.

53. de Almeida FM, Marques SA, Ramalho Bdos S, et al. Chronic spinal cord lesions respond positively to tranplants of mesenchymal stem cells. Restor Neurol Neurosci 2015; 33: 43-55.

54. Tsuda H, Yamahara K, Ishikane S, et al. Allogenic fetal membrane-derived mesenchymal stem cells contribute to renal repair in experimental glomerulonephritis. Am J Physiol Renal Physiol 2010; 299: F1004-13.

55. Zimmermann JA, Mcdevitt TC. Pre-conditioning mesenchymal stromal cell spheroids for immunomodulatory paracrine factor secretion. Cytotherapy 2014; 16: 331-45.

56. Zgheib A, Pelletier-Bonnier É, Levros LC Jr, Annabi B. Selective JAK/ STAT3 signalling regulates transcription of colony stimulating factor-2 and -3 in Concanavalin-A-activated mesenchymal stromal cells. Cytokine 2013; 63: 187-93.

57. Gong L, Zhao Y, Zhang Y, et al. The macrophage polarization regulates MSC osteoblast differentiation in vitro. Ann Clin Lab Sci 2016; 46: 65-71.

58. Fernandes TJ, Hodge JM, Singh PP, et al. Cord blood-derived macrophage-lineage cells rapidly stimulate osteoblastic maturation in mesenchymal stem cells in a glycoprotein-130 dependent manner. PLoS One 2013; 8: e73266.

59. Sesia SB, Duhr R, Medeiros da Cunha C, et al. Anti-inflammatory/ tissue repair macrophages enhance the cartilage forming capacity of human bone marrow-derived mesenchymal stromal cells. J Cell Physiol 2015; 230: 1258-69.

60. Ben-Mordechai T, Holbova R, Landa-Rouben N, et al. Macrophage subpopulations are essential for infarct repair with and without stem cell therapy. J Am Coll Cardiol 2013; 62: 1890-901.

61. Li O, Sun W, Wang X, Zhang K, Xi W, Gao P. Skin-derived mesenchymal stem cells alleviate atherosclerosis via modulating macrophage function. Stem Cells Transl Med 2015; 4: 1294-301.

62. Braza F, Dirou S, Forest V, et al. Mesenchymal stem cells induce suppressive macrophages through phagocytosis in a mouse model of asthma. Stem Cells 2016; 34: 1836-45.

63. Lee S, Szilagyi E, Chen L, et al. Activated mesenchymal stem cells increase wound tensile strength in aged mouse model via macrophages. J Surg Res 2013; 181: 20-4.

64. Dayan V, Yannarelli G, Billia F, Filomeno P, Wang XH, Davies JE, Keating A. Mesenchymal stromal cells mediate a switch to alternatively activated monocytes/macrophages after acute myocardial in farction. Basic Res Cardiol 2011; 106: 1299-310.

65. Eliadou E, Day AS, Thompson-Fawcett MW, et al. New Zealand society of gastroenterology guidelines for the management of refractory ulcerative colitis. N Z Med J 2015; 128: 63-76.

66. Okamoto R, Watanabe M. Investigating cell therapy for inflammatory bowel disease. Expert Opin Biol Ther 2016; 16: 1015-23.

67. Dave M, Jaiswal P, Cominelli F. Mesenchymal stem/stromal cell therapy for inflammatory bowel disease: an updated review with maintenance of remission. Curr Opin Gastroenterol 2017; 33: 59-68.

68. Jergens A, Young J, Moore D, et al. Bcl-2/caspase 3 mucosal imbalance favors t cell resistance to apoptosis in dogs with inflammatory bowel disease. Vet Immunol Immunopathol 2014; 158: 167-74.

69. Chen QQ, Yan L, Wang CZ, et al. Mesenchymal stem cells alleviate TNBS-induced colitis by modulating inflammatory and autoimmune responses. World J Gastroenterol 2013; 19: 4702-17. 
70. Grégoire C, Lechanteur C, Briquet A, et al. Review article: mesenchymal stromal cell therapy for inflammatory bowel diseases. Aliment Pharmacol Ther 2017; 45: 205-21.

71. Zuo D, Liu X, Shou Z, et al. Study on the interactions between transplanted bone marrow-derived mesenchymal stem cells and regulatory $t$ cells for the treatment of experimental colitis. Int J Mol Med 2013; 32: 1337-44.

72. González MA, Gonzalez-Rey E, Rico L, et al. Adipose-derived mes enchymal stem cells alleviate experimental colitis by inhibiting inflammatory and autoimmune responses. Gastroenterology 2009; 136: 978-89.

73. Dhere T, Copland I, Garcia M, et al. The safety of autologous and metabolically fit bone marrow mesenchymal stromal cells in medically refractory Crohn's disease - a phase 1 trial with three doses. Aliment Pharmacol Ther 2016; 44: 471-81.

74. Ko IK, Kim BG, Awadallah A, et al. Targeting improves MSC treatment of inflammatory bowel disease. Mol Ther 2010; 18: 1365-72.

75. Chen Z, He X, He X, et al. Bone marrow mesenchymal stem cells ameliorate colitis-associated tumorigenesis in mice. Biochem Biophys Res Commun 2014; 450: 1402-8.

76. Duijvestein M, Molendijk I, Roelofs H, et al. Mesenchymal stromal cell function is not affected by drugs used in the treatment of inflammatory bowel disease. Cytotherapy 2011; 13: 1066-73.

77. Xing Y, Chen X, Cao Y, et al. Expression of wnt and notch signaling pathways in inflammatory bowel disease treated with mesenchymal stem cell transplantation: evaluation in a rat model. Stem Cell Res Ther 2015; 6: 101

78. Wang WQ, Dong K, Zhou L, et al. II-37b gene transfer enhances the therapeutic efficacy of mesenchymal stromal cells in dss-induced colitis mice. Acta Pharmacol Sin 2015; 36: 1377-87.

79. Chinnadurai R, Ng S, Velu V, et al. Challenges in animal modelling of mesenchymal stromal cell therapy for inflammatory bowel disease. World J Gastroenterol 2015; 21: 4779-87.

80. Steinbach EC, Plevy SE. The role of macrophages and dendritic cells in the initiation of inflammation in IBD. Inflamm Bowel Dis 2014; 20: 166-75.

81. Agier J, Efenberger M, Brzezińska-Błaszczyk E. Cathelicidin impact on inflammatory cells. Cent Eur J Immunol 2015; 40: 225-35.

82. Lissner D, Schumann M, Batra A, et al. Monocyte and M1 macrophage-induced barrier defect contributes to chronic intestinal inflammation in IBD. Inflamm Bowel Dis 2015; 21: 1297-305.

83. Wang C, Chen J, Sun L, et al. TGF- $\beta$ signaling-dependent alleviation of dextran sulfate sodium-induced colitis by mesenchymal stem cell transplantation. Mol Biol Rep 2014; 41: 4977-83.

84. Fan H, Zhao G, Liu L, et al. Pre-treatment with IL-1 $\beta$ enhances the efficacy of msc transplantation in dss-induced colitis. Cell Mol Immunol 2012; 9: 473-81.

85. Simovic Markovic B, Nikolic A, Gazdic M, et al. Pharmacological inhibition of gal-3 in mesenchymal stem cells enhances their capacity to promote alternative activation of macrophages in dextran sulphate sodium-induced colitis. Stem Cells Int 2016; 2016: 2640746.

86. Mao F, Wu Y, Tang X, et al. Human umbilical cord mesenchymal stem cells alleviate inflammatory bowel disease through the regulation of 15-LOX-1 in macrophages. Biotechnol Lett 2017; 39: 929-38.

87. Johnson CM, Wei C, Ensor JE, et al. Meta-analyses of colorectal cancer risk factors. Cancer Causes Control 2013; 24: 1207-22.

88. Kulaylat MN, Dayton MT. Ulcerative colitis and cancer. J Surg Oncol 2010; 101: 706-12.

89. Eaden JA, Abrams KR, Mayberry JF. The risk of colorectal cancer in ulcerative colitis: A meta-analysis. Gut 2001; 48: 526-35.

90. Canavan C, Abrams KR, Mayberry J. Meta-analysis: Colorectal and small bowel cancer risk in patients with Crohn's disease. Aliment Pharmacol Ther 2006; 23: 1097-104.

91. Ferlay J, Soerjomataram I, Dikshit R, et al. Cancer incidence and mortality worldwide: sources, methods and major patterns in GLOBOCAN 2012. Int J Cancer 2015; 136: E359-86.

92. Brenner H, Kloor M, Pox CP. Colorectal cancer. Lancet 2014; 383: 1490-502.

93. Owens BM. Inflammation, Innate Immunity, and the Intestinal Stromal Cell Niche: Opportunities and Challenges. Front Immunol 2015; 6: 319.
94. Cook J, Hagemann T. Tumour-associated macrophages and cancer. Curr Opin Pharmacol 2013; 13: 595-601.

95. Mantovani A. MSCs, macrophages, and cancer: a dangerous ménage-à-trois. Cell Stem Cell 2012; 11: 730-32.

96. Brzozowa M, Michalski M, Wyrobiec G, et al. The role of Snail1 transcription factor in colorectal cancer progression and metastasis. Contemp Oncol (Pozn) 2015; 19: 265-70.

97. Ryan AE, Colleran A, O'Gorman A, et al. Targeting colon cancer cell NF-kB promotes an anti-tumour M1-like macrophage phenotype and inhibits peritoneal metastasis. Oncogene 2015; 34: 1563-74.

98. Cho DI, Kim MR, Jeong HY, Jeong HC, Jeong MH, Yoon SH, Kim YS, Ahn Y. Mesenchymal stem cells reciprocally regulate the M1/M2 balance in mouse bone marrow-derived macrophages. Exp Mol Med 2014; 46: e70.

\section{Address for correspondence}

\section{Wenrong Xu}

School of Medical Science and Laboratory Medicine

Jiangsu University

301 Xuefu Road, Zhenjiang

Jiangsu 212013, China

e-mail: icls@ujs.edu.cn

\section{Xu Zhang}

School of Medical Science and Laboratory Medicine

Jiangsu University

301 Xuefu Road, 212013 China

e-mail:xuzhang@ujs.edu.cn.

Submitted: 2.05 .2017

Accepted: 17.05 .2017 